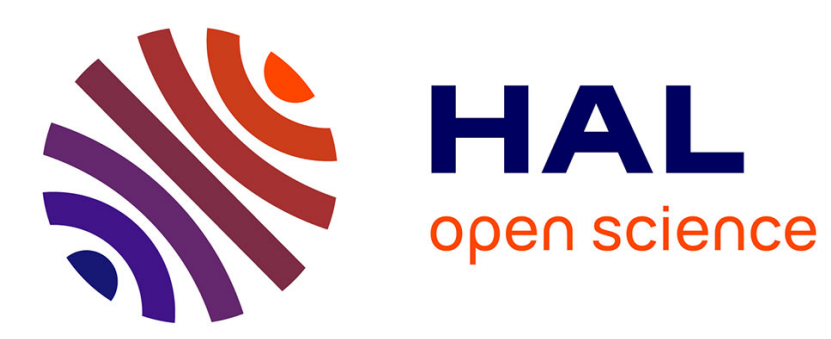

\title{
Augmentation de la densité de courant critique par dopage de YBCO texturé au BaSnO3
}

M. Lepropre, I. Monot, M. Delamare, M. Hervieu, Ch. Simon, J. Provost, G. Desgardin, B. Raveau

\section{- To cite this version:}

M. Lepropre, I. Monot, M. Delamare, M. Hervieu, Ch. Simon, et al.. Augmentation de la densité de courant critique par dopage de YBCO texturé au BaSnO3. Journal de Physique III, 1994, 4 (11), pp.2205-2212. 10.1051/jp3:1994272 . jpa-00249255

\section{HAL Id: jpa-00249255 https://hal.science/jpa-00249255}

Submitted on 1 Jan 1994

HAL is a multi-disciplinary open access archive for the deposit and dissemination of scientific research documents, whether they are published or not. The documents may come from teaching and research institutions in France or abroad, or from public or private research centers.
L'archive ouverte pluridisciplinaire HAL, est destinée au dépôt et à la diffusion de documents scientifiques de niveau recherche, publiés ou non, émanant des établissements d'enseignement et de recherche français ou étrangers, des laboratoires publics ou privés. 


\title{
Augmentation de la densité de courant critique par dopage de YBCO texturé au $\mathrm{BaSnO}_{3}$
}

\author{
M. Lepropre, I. Monot, M. P. Delamare, M. Hervieu, Ch. Simon, J. Provost. \\ G. Desgardin et B. Raveau
}

Laboratoire CRISMAT ISMRA/CNRS et Université de Caen, Bd. Maréchal Juin, 14050 Caen Cedex. France

(Reçu le 3 février 1994, révisé le 25 avril 1994, accepté le 11 mai 1994)

\begin{abstract}
Résumé. - Nous avons mesuré la densité de courant critique de transport (courant continu) $J_{c}$, dans une céramique texturée $\mathrm{YBaCuO}$ dopée au $\mathrm{BaSnO}_{3}$. Elle présente des valeurs de $J_{\mathrm{c}}(77 \mathrm{~K})$ de $7,1 \times 10^{4} \mathrm{~A} \mathrm{~cm}^{-2}$ en champ magnétique nul et de $1,1 \times 10^{4} \mathrm{~A} \mathrm{~cm}^{-2}$ sous $20 \mathrm{~T}$. Une étude systématique de cette céramique a été entreprise. La microstructure et la nanostructure observées respectıvement en microscopie électronique à balayage SEM et en microscopie à haute résolution HREM ont été corrélées avec les mesures de $J_{c}$. On observe dans cette céramique une microstructure texturée contenant de nombreuses inclusions de phase verte (taille $\leqslant 10 \mu \mathrm{m}$ ) et un très faible nombre d'inclusions de $\mathrm{BaSnO}_{3}$ (taille $<1 \mu \mathrm{m}$ ). Les valeurs de $J_{\mathrm{c}}$ de transport se révèlent être assez dispersées $\left(2,5 \times 10^{3}\right.$ à $\left.7,1 \times 10^{4} \mathrm{~A} \mathrm{~cm}^{2}\right)$. Des mesures de $J_{\mathrm{c}}$ par méthode magnétique ont été effectuées. Le résultat de ces mesures suggère la présence de microfissures dans certaines régions du matériau causant alors une chute dramatique du $J_{\mathrm{c}}$ de transport dans l'échantillon correspondant. D'autre part, les observations HREM démontrent que les défauts étendus recensés ne peuvent pas être tenus pour principaux responsables du piégeage des vortex dans ce type de matériau. L'éventuelle inhomogénéité à l'échelle nanostructurale ne semble pas influencer la densité de courant critique. Le piégeage seraıt donc principalement gouverné par la microstructure lamellaire et non par la présence d'inclusions de $\mathrm{BaSnO}_{3}$.
\end{abstract}

\begin{abstract}
We have performed critical current density measurements (DC current) on melt textured $\mathrm{YBaCuO}$ doped with $\mathrm{BaSnO}_{3}$ which exhibits transport values at $77 \mathrm{~K}$ as high as $7.1 \times 10^{4} \mathrm{~A} \mathrm{~cm}^{-2}$ in zero field and $1.1 \times 10^{4} \mathrm{~A} \mathrm{~cm}^{-2}$ at $20 \mathrm{~T}$. A systematic study of this ceramic has been carried out using SEM and HREM observations in correlation with $J_{c}$ measurements. A textured microstructure is observed containing numerous green phase inclusions (size $\leqslant 10 \mu \mathrm{m}$ ) as well as a few numbers of $\mathrm{BaSnO}_{3}$ inclusions (size $<1 \mu \mathrm{m}$ ). It has been found that transport $J_{\mathrm{c}}$ data are distributed over a wide range $\left(2.5 \times 10^{3}-7.1 \times 10^{4} \mathrm{~A} \mathrm{~cm}^{-2}\right)$. Nevertheless, magnetic $J_{\mathrm{c}}$ measurements suggest that cracks of the order of several microns may appear in some regions of the material leading to a dramatic decrease of transport $J_{c}$ for the corresponding sample. On the other hand. HREM observations demonstrate that extended defects cannot be considered as the major factor for vortex pinning in textured 123 material. The presence or the absence of inhomogeneity at the nanoscale does not seem to influence the critical current density. Finally. it has been determined that pinning can be ascribed to lamellar microstructure rather than to $\mathrm{BaSnO}_{3}$ inclusions.
\end{abstract}




\section{Introduction.}

Le composé YBaCuO supraconducteur à $92 \mathrm{~K}$ est reconnu comme matériau potentiel pour les applications puisqu il est possible de l'utiliser à la température de l'azote liquide et que sa synthèse est assez facile à mettre en œuvre. Cependant, pour les applications en électrotechnique, des valeurs de densité de courant critique de $10^{4}$ à $10^{5} \mathrm{~A} \mathrm{~cm}^{-2}$ sous un champ magnétique de 1 Tesla sont généralement requises. Les valeurs de $J_{\mathrm{c}}$ dépendent fortement de la microstructure de la céramique. Le nombre de fissures doit être minimisé et le nombre de joints de grains transverses doit être minimal. Des procédés de fusion et de croissance orientée de grains, ont été développés $[1,2]$ dans le but de texturer le matériau et donc d'augmenter les valeurs de $J_{\mathrm{c}}$. La technique « Melt Textured Growth » (MTG) permet en effet de préparer des matériaux dont le $J_{\mathrm{c}}$ peut atteindre $3,5 \times 10^{4} \mathrm{~A} \mathrm{~cm}^{-2}$ à $77 \mathrm{~K}$ sous champ nul et $2,6 \times 10^{4} \mathrm{~A} \mathrm{~cm}^{-2}$ sous $7 \mathrm{~T}$ [3]. Ces valeurs, quoique très prometteuses sont encore d'un ordre de grandeur inférieures aux valeurs souhaitées pour la plupart des applications et sont difficiles à reproduire de façon homogène sur des échantillons longs. Des résultats encourageants ont été par ailleurs obtenus en densité de courant critique à bas champ dans des céramiques texturées de type $\mathrm{YBaCuO}$ dopées au $\mathrm{BaSnO}_{3}[4.5]$, mais aucune valeur de $J_{c}$ à haut champ n'a encore été mesurée. De plus, aucune observation en microscopie électronique n'a été présentée. Nous nous sommes donc proposés d'étudier systématiquement les céramiques de type YBaCuO texturées (MTG) dopées au $\mathrm{BaSnO}_{3}$, au moyen d'observations en microscopie électronique SEM et HREM, et de corréler les résultats de ces observations avec les mesures de densité de courant critique de transport sous champ à $77 \mathrm{~K}$.

\section{Procédure expérimentale.}

La poudre $\mathrm{BaSnO}_{3}$ préparée par réaction à l'état solide a été ajoutée à la poudre $\mathrm{YBa}_{2} \mathrm{Cu}_{3} \mathrm{O}_{7-\delta}$ (123) Rhône Poulenc dans les proportions molaires de 4,5\% (2\% en masse). Après mélange et broyage en attritor, dans l'alcool, assurant une excellente homogénéité du mélange, la poudre séchée a été pressée, isostatiquement à $300 \mathrm{MPa}$ sous forme de barreaux $\left(50 \times 10 \times 7 \mathrm{~mm}^{3}\right)$ et fritté à $940^{\circ} \mathrm{C}$ pendant $12 \mathrm{~h}$ sous flux d'oxygène. Les barreaux obtenus ont alors été placés sur des supports de phase verte frittée $\left(\mathrm{Y}_{2} \mathrm{BaCuO}_{5}\right.$ dite « $\left.211 »\right)$ qui a été choisie pour l'identité des éléments de la phase avec 123 et pour la faible différence des coefficients de dilatation thermique de ces deux phases [6]. Ceci permet la formation d'une interface continue sans fissure. Le procédé MTG a été réalisé dans un four horizontal présentant deux zones de chauffage avec un plateau chaud à $1200^{\circ} \mathrm{C}$ et une zone où règne un gradient thermique de $20^{\circ} \mathrm{C} . \mathrm{cm}^{-1}$ (Fig. 1). Après une très courte période à haute température pour obtenir une fusion partielle résultant de la décomposition péritectique de 123 , l'échantillon a été déplacé vers le gradient puis refroidi lentement $\left(1^{\circ} \mathrm{C} / \mathrm{h}\right)$ dans cette zone sous flux d'oxygène.

\section{Mesures et observations.}

Dans le but de comprendre les effets de la microstructure et de la nanostructure sur le courant critique, une série de mesures électriques et magnétiques ont été réalisées sur cinq barreaux différents de dimensions typiques $\left(0,1 \times 0,1 \times 0,8 \mathrm{~mm}^{3}\right)$, découpés dans le même matériau massif 123/ $\mathrm{BaSnO}_{3}$. Les résultats de ces mesures ont été corrélés avec les observations en SEM et en HREM faites sur ces 5 échantillons.

Cette céramique présente une transition supraconductrice étroite à $91 \mathrm{~K}$ mesurée au susceptomètre $\mathrm{AC}$ avec un champ alternatif appliqué parallèlement au plan $(a b) \mathrm{de} 10 \mathrm{G}$ $\left(\Delta T_{\imath}=1,5 \mathrm{~K}\right)$. 


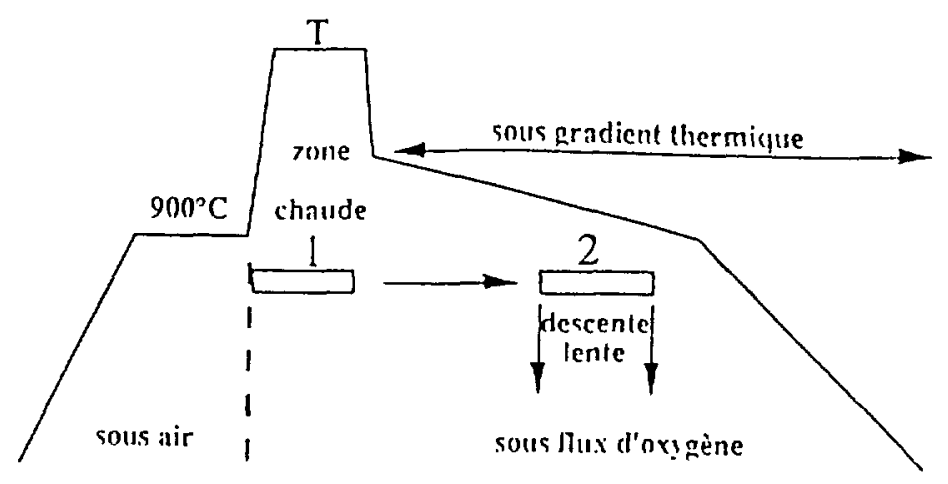

Fig. 1. - Profil thermique présentant les deux étapes de la synthèse du matériau texturé.

[Thermal profile showing the two steps of the texturing process.]

Les observations par SEM des différents échantillons confirment la qualité de la texture comme l'illustre la figure 2. Le système d'analyse TRACOR associé au microscope SEM a permis d'identifier les différentes inclusions visibles sur cette figure. Seules les trois inclusions marquées d'une flèche correspondent au $\mathrm{BaSnO}_{3}$ tandis que les autres, habituellement observées dans YBCO texturé, correspondent à de la phase verte. (La distinction de ces deux types d'inclusions est difficile à faire sur cette reproduction à cause de son faible contraste).

MESURES DE COURANTS CRITIQUES DE TRANSPORT. - Les mesures de $J_{c}$ de transport ont été réalisées par la méthode classique 4 pointes. Nous avons adopté un critère de champ électrique

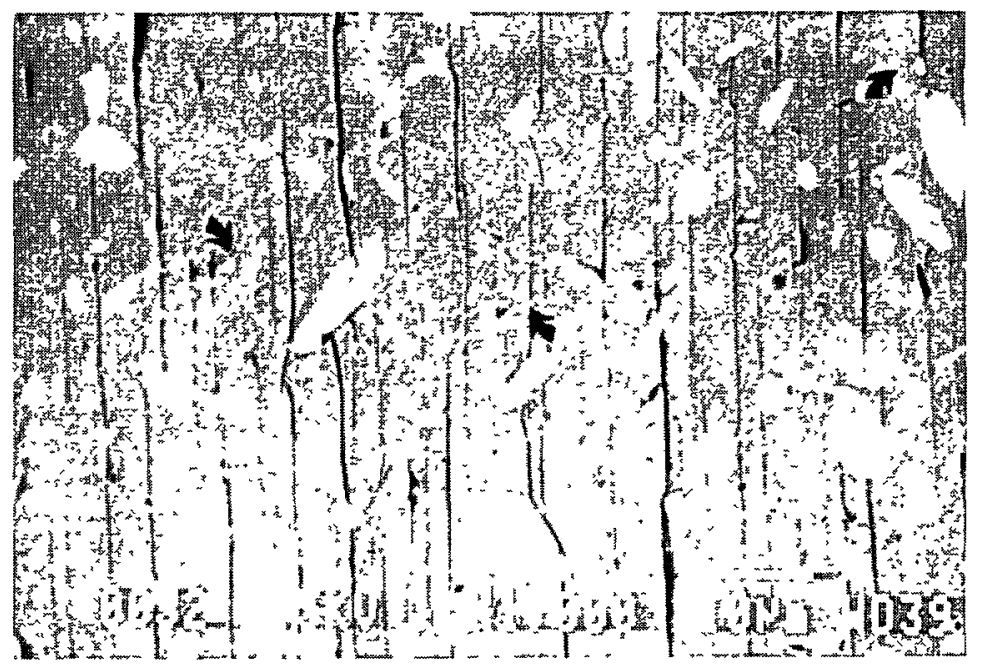

Fig. 2. - Micrographie SEM de la céramique texturée $123 / \mathrm{BaSnO}_{3}$ montrant la microstructure en lamelles de 5-7 $\mu \mathrm{m}$. Les inclusions dont la talle varie d'environ 1 à $10 \mu \mathrm{m}$ sont des particules de 211 . Les petites inclusions (taille $<1 \mu \mathrm{m}$ ) indiquées par des flèches sont des partıcules de $\mathrm{BaSnO}_{3}$.

[The SEM micrograph of the textured ceramic $123 / \mathrm{BaSnO}_{3}$ shows the $5-7 \mu \mathrm{m}$ lamellar microstructure. The 211 inclusions are $1-10 \mu \mathrm{m}$ large whereas the smaller ones (size $<1 \mu \mathrm{m}$ ) indicated by curved arrows are $\mathrm{BaSnO}_{\text {; particles.] }}$ 
de $5 \mu \mathrm{V} \mathrm{cm}^{-1}$ compte tenu de la taille des échantillons et de la résolution du voltmètre. Le courant continu a été appliqué dans la direction des plans $\left[\mathrm{CuO}_{2}\right]_{\infty}$ (plans $a b$ ). Les résultats relatifs aux cinq échantillons sont résumés dans le tableau 1.

Tableau 1. - Densité de courant critique à $77 \mathrm{~K}$ mesurée en transport ou magnétiquement sur les cinq échantillons découpés dans le même barreau de matériau massif $123 / \mathrm{BaSnO}_{3}$. La qualité de la nanostructure correspondante observée en HREM est indiquée dans la dernière colonne.

[Critical current density at $77 \mathrm{~K}$ measured on the five samples cut out of the same bulk material $123 / \mathrm{BaSnO}_{3}$, by transport method or deduced from magnetization cycles. The corresponding nanostructure quality observed by HREM is also indicated.]

\begin{tabular}{|c|c|c|c|}
\hline Echantillon & \multicolumn{2}{|c|}{$J_{\mathrm{c}}\left(10^{3} \mathrm{~A} \mathrm{~cm}^{2}\right)$} & $\begin{array}{c}\text { HREM } \\
\text { nanostructure }\end{array}$ \\
\hline & Transport & SQUID $(B=0)$ & \\
\hline 1 & $56 \quad(B=0)$ & - & désordonnée \\
2 & $2,5(B=0)$ & - & régulière \\
3 & $5 \quad(B=0)$ & $50-70$ & régulière \\
4 & $71 \quad(B=0)$ & $50-70$ & régulière \\
5 & $11 \quad(B=20 \mathrm{~T})$ & - & - \\
\hline
\end{tabular}

La figure 3 illustre la très grande différence de comportement entre les courbes $J_{c}=f(B)$ des échantillons 2 et 4 soumis à un champ magnétique à peu près parallèle aux plans $(a b)$.

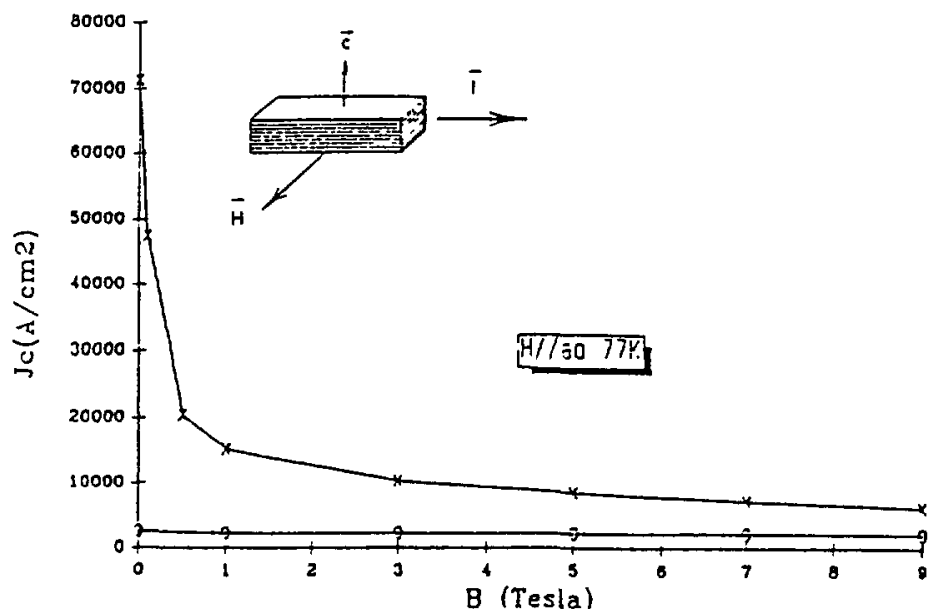

Fig. 3. - $J_{c}$ de transport à $77 \mathrm{~K}$ avec un champ magnétique à peu près parallèle aux plans $(a b)$. Le courant circule dans ces plans et perpendiculairement au champ. O échantillon 2 ; X échantillon 4. La décroissance de $j_{\iota}(B)$ de l'échantillon 4 au-dessus de 1 Tesla provient d'une légère désorientation du plan $a b$ par rapport au champ.

[Transport $J_{c}$ at $77 \mathrm{~K}$ under external magnetic field applied close to the $(a b)$ planes. Current flows in these planes, normaly to the field. O sample $2 ; X$ sample 4 . The $j_{c}(B)$ decrease of sample 4 above 1 Tesla is due to a slight misalignment of the field with respect to the $a b$ plane.] 
La dispersion des valeurs de $J_{c}$ peut être due à une inhomogénéité au niveau de la nanostructure ou simplement à la présence de microfissures pouvant apparaître dans certaines régions du matériau et conduisant à une chute dramatique de $J_{c}$ dans les échantillons correspondants. Dans le but de vérifier ces hypothèses, nous avons effectué des mesures magnétiques et observé ces échantillons en HREM.

MESURES DE COURANTS CRITIQUES D'AIMANTATION. - Des mesures de cycles d'hystérésis magnétique à $77 \mathrm{~K}$ ont été réalisées à l'aide d'un magnétomètre à SQUID (champ parallèle à l'axe c), sur des échantillons présentant des $J_{c}$ de transport très différents (échantillons 3 et 4 ). La densité de courant critique déduite de ces cycles a été calculée au moyen du modèle de Bean modifié [7] et les résultats sont reportés sur la figure 4.

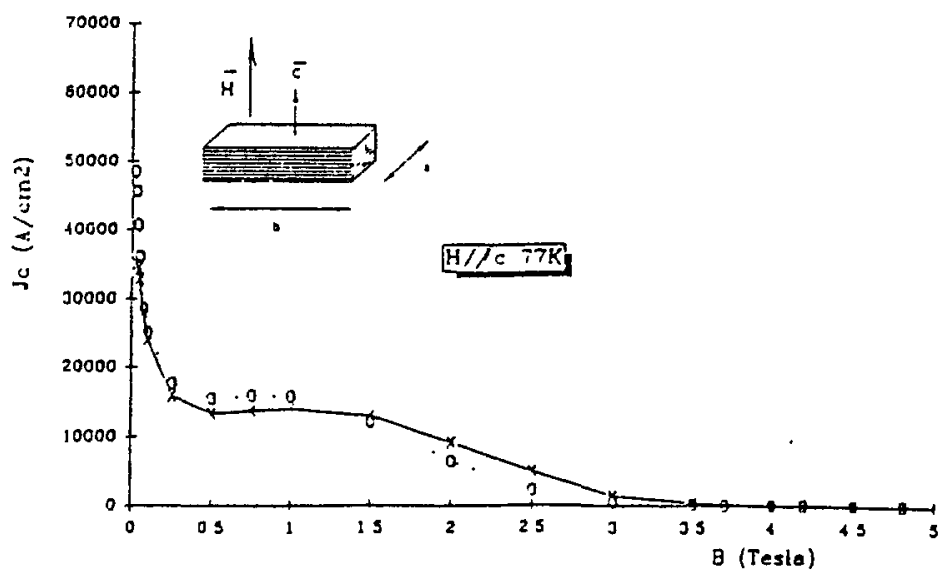

Fig. 4. - Densités de courant critique des échantıllons 4 et 3 calculées à partir des cycles d'aımantation à $77 \mathrm{~K}$ en champ parallèle à l'axe $c$. X échantillon 4 ; O échantillon 3.

[Critical current densities of samples 4 and 3 deduced from the magnetization cycles (field parallel to the c-axis). X sample 4 ; O sample 3.]

Cette figure montre clairement que les valeurs et les comportements des $J_{\mathrm{c}}$ d'aimantation sont pratiquement identiques pour ces deux échantillons $\left(5 \times 10^{4}\right.$ et $7 \times 10^{4} \mathrm{~A} \mathrm{~cm}^{-2}$ pour $B=0$ ) alors que les valeurs de $J_{\mathrm{c}}$ mesurées en transport sont très différentes. On peut donc en déduire que le faible $J_{c}$ de transport de l'échantillon 3 serait plutôt dû à la présence de microfissures. Au contraire, celles-ci semblent absentes dans l'échantillon 4.

OBSERVATIONS EN MICROSCOPIE ÉLECTRONIQUE HREM. - Les échantillons 3 et 4 présentent une nanostructure dont la périodicité et le contraste réguliers révèlent l'absence de défauts à cette échelle, comme en témoigne la figure 5.

Cette observation est en parfait accord avec celle des mesures magnétiques effectuées sur ces échantillons. D'autre part, l'échantillon 1 (fort $J_{c}$ de transport) présente une nanostructure très désordonnée comme le révèle la figure 6.

On observe sur cette photographie la présence des phases « 124 » et « 134 » très localisées, d'intercalations de segments $\mathrm{CuO}$ additionnels (A), de substitutions atomiques et de mécanismes de cisaillement (B), de variations locales d'orientation de groupes $\mathrm{CuO}_{4}(\mathrm{C})$ et de superdislocations à la frontière des phases localisées « $124 »$ et « $134 »$ (D). 


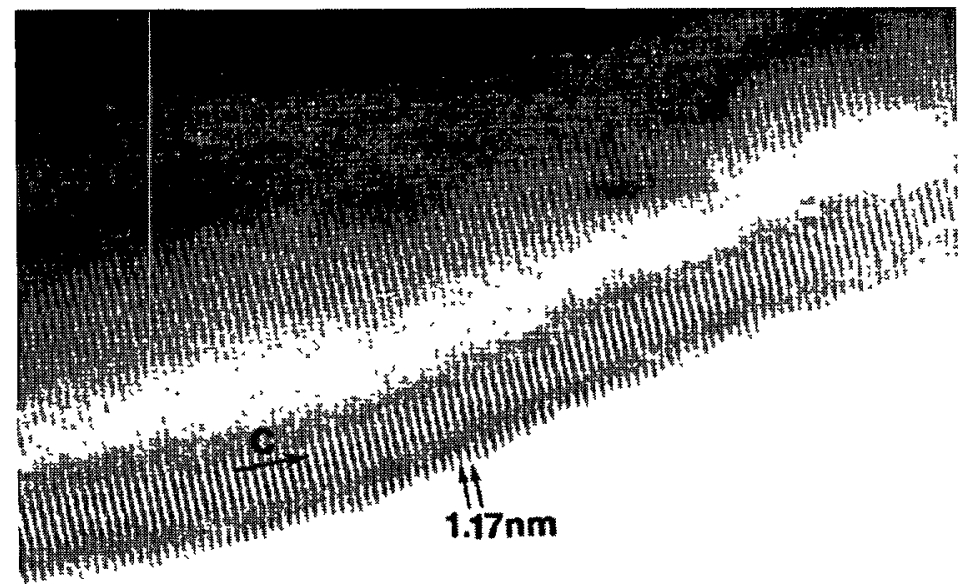

[100]

Fig. 5. - La grande régularité des franges espacées de 11,7 de la micrographie HREM des échantillons 3 et 4 met en évidence l'absence de défauts à l'échelle nanostructurale.

[The HREM micrograph of samples 3 and 4 shows a high regularity of the fringes spaced by $11.7 \AA$ and emphasizes the absence of nanostructural faults.]

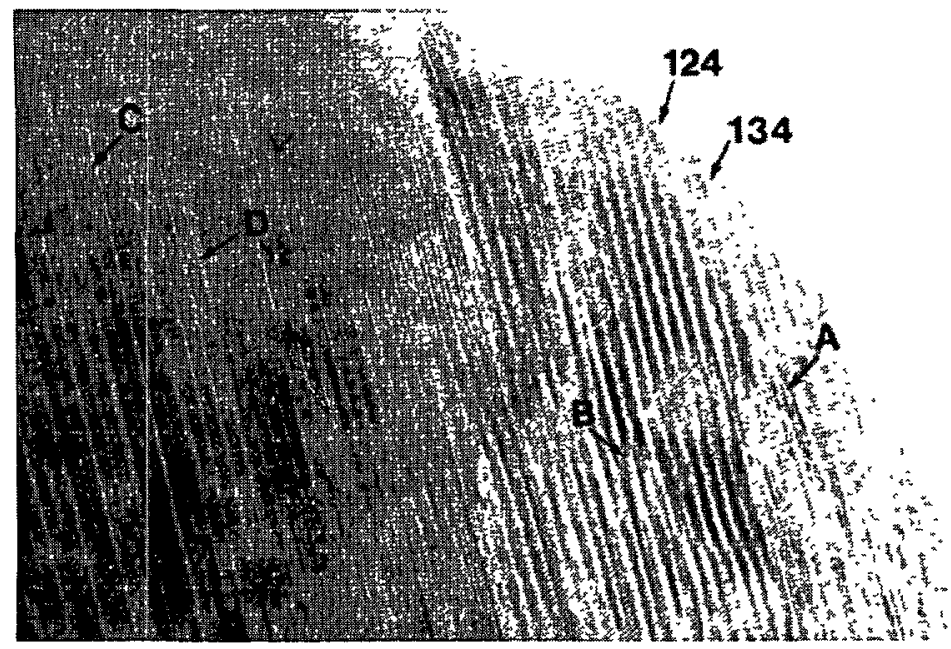

Fig. 6. - Mıcrographie HREM de l'échantillon 1 où apparaissent intercalations, superdislocations et mécanısmes de cisaillement.

[The sample I HREM micrograph evıdences intercalations, superdislocations and shearing mechanisms.]

Par conséquent, la comparaison des échantillons 1 et 4 montre qu en dépit de leurs valeurs de $J_{\mathrm{c}}$ de transport proches et très élevées. ils présentent des nanostructures très différentes. Ceci montre que les défauts étendus à cette échelle ne peuvent pas être considérés comme les principaux responsables du piégeage des vortex dans le matériau « 123 » en champ faible. 
COMPORTEMENT SOUS CHAMP. - Le comportement sous champ de ces échantillons est très semblable à celui des échantillons sans $\mathrm{BaSnO}_{3}$ [9]. La comparaison de la figure 3 et de la figure 4 montre que le courant critique diminue assez vite de façon assez isotrope avec le champ appliqué jusqu'à 1 Tesla environ. Il devient alors très anisotrope : il s'écroule si le champ est appliqué selon l'axe $c$ et est presque indépendant du champ si il est appliqué dans les plans $(a b)$. A fort champ, il subsiste un pic très clair autour des plans ( $a b)$ comme le montre la figure 7.

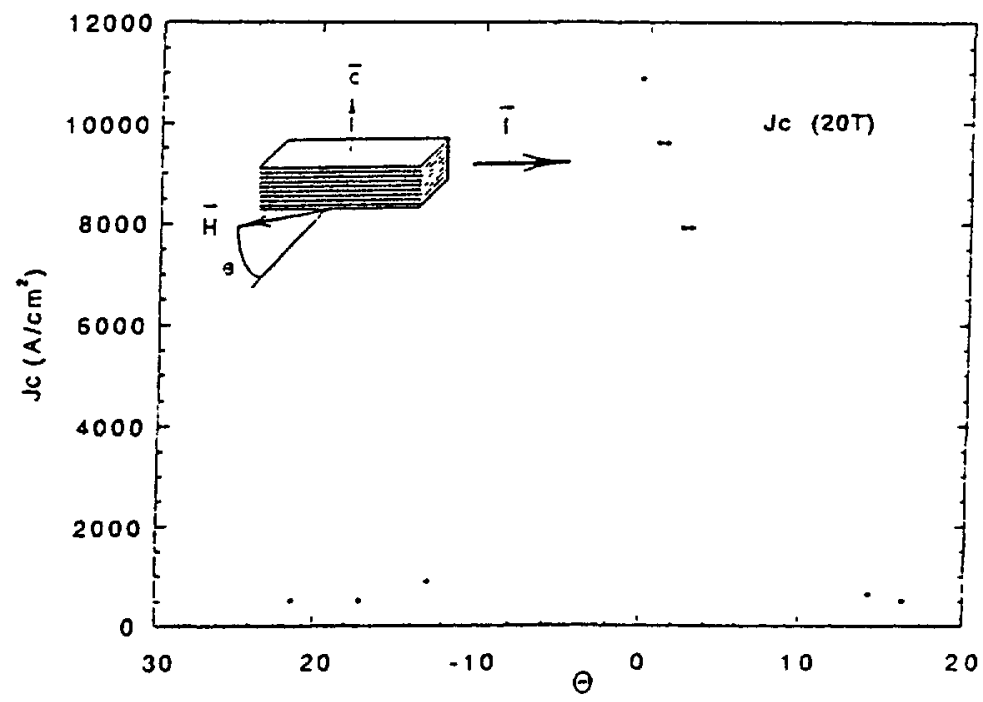

Fig. 7. - Densité de courant critique en transport à $77 \mathrm{~K}$ en fonction de l'angle de rotation $\theta$ du champ par rapport aux plans $(a b)$ de l'échantillon 5. Le champ est perpendiculaire au courant et parallèle aux plans $(a b)$ lorsque $\theta=0$.

[Critical current density at $77 \mathrm{~K}$ of sample 5 versus rotation angle around $(a b)$ planes. The field is normal to the current and is located within these planes when $\theta=0$.]

Une comparaison entre les résultats obtenus sur des monocristaux [10] d'épaisseur variable et les matériaux texturés avec ou sans $\mathrm{BaSnO}_{3}$ montre que la densité de courant critique en champ faible est inversement proportionnelle à l'épaisseur des échantillons (ou des lamelles dans le cas des matériaux texturés à structure lamellaire), ce qui est très en faveur d'un modèle de piégeage par les inhomogénéités de surface [8]. Dans ce cadre, l'amélioration des densités de courant critique en champ faible par l'addition de $\mathrm{BaSnO}_{3}$ pourrait provenir d'une périodicité plus faible de la structure lamellaire (un facteur 2 environ).

Deux modèles sont proposés pour comprendre le comportement en champ fort. Le premier fait appel au piégeage intrinsèque par la nanostructure [11] et devrait être insensible à la présence de $\mathrm{BaSnO}_{3}$. Or, $\mathrm{BaSnO}_{3}$ semble modifier ce piégeage $\left(\approx 1,1 \times 10^{4} \mathrm{~A} \mathrm{~cm}^{-2}\right.$ au lieu de $2,8 \times 10^{4} \mathrm{~A} \mathrm{~cm}^{-2}$ [3].) Le second modèle fait appel au piégeage par la présence des surfaces des lamelles de la microstructure [12], mais n'apporte pas encore de prédictions précises sur le comportement en champ magnétique appliqué. Des mesures complémentaires seront nécessaires pour distinguer entre les deux modèles et pour progresser dans l'optimisation du matériau en champ fort. 


\section{Conclusions.}

En conclusion, le dopage de la céramique texturée $\mathrm{YBaCuO}$ par $\mathrm{BaSnO}_{3}$ semble diminuer les contraintes internes, notamment lors de la transition quadratique-orthorhombique. Ce dopage procure une microstructure en cristaux 123 lamellaires plus minces $(\approx 7 \mu \mathrm{m})$. Cette caractéristique semble favoriser le piégeage des vortex en champ faible, et des valeurs élevées de $J_{\mathrm{c}}$ en résultent. Il devrait donc être possible d'améliorer encore la densité de courant critique en champ faible en diminuant l'épaisseur des lamelles à l'échelle microstructurale. L'influence de l'addition de $\mathrm{BaSnO}_{3}$ sur le comportement en champ fort reste à étudier plus précisément.

\section{Remerciements.}

Nous remercions l'équipe de R. Tournier du laboratoire MATFORMAG (Grenoble) pour les mesures angulaires de $J_{\mathrm{c}}$ présentées à la figure 7 .

\section{Bibliographie}

[1] Jin S. et al., High critical currents in Y-Ba-Cu-O superconductors, Appl. Phys Lett 52 (1988) 2074.

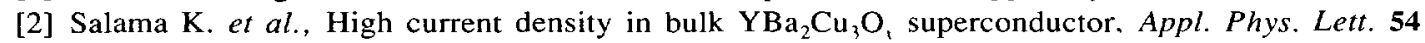
(1989) 2352.

[3] Monot I. et al., High performance in a bulk melt-textured $\mathrm{YBa}_{2} \mathrm{Cu}_{3} \mathrm{O}_{7-\delta}$ ceramic, Supercond. Sci. Technol. 5 (1992) 712.

[4] McGinn P. et al., Microstructure and critical current density of zone melt textured $\mathrm{YBa}_{2} \mathrm{Cu}_{3} \mathrm{O}_{6+} / \mathrm{Y}_{2} \mathrm{BaCuO}_{5}$ with $\mathrm{BaSnO}_{3}$ additions. Appl. Phys. Lett. 59 (1991) 120.

[5] McGinn P. et al., Microstructure and magnetic properties of zone melt textured $\mathrm{YBa}_{2} \mathrm{Cu}_{3} \mathrm{O}_{6+}$, with $\mathrm{BaSnO}_{3}$ additions, Physica $\mathrm{C} 183$ (1991) 51.

[6] Hrncir K. et al., Polycrystalline substrates for $\mathrm{YBa}_{2} \mathrm{Cu}_{3} \mathrm{O}_{7-\delta} / \mathrm{YBaCuO}$ films, Proc. First European Ceramic Conf., Euroceramics 2 (Elsevier Applied Science, 1989) p. 461.

[7] Chen D. X. and Goldfarb R. B., Kım model for magnetization of type II superconductors, J. Appl. Phys. 66 (1989) 2489.

[8] Hocquet T. et al., Continuum theory of the mixed-state and surface Joule effects in type II superconductors, Phys. Rev. B 36 (1992) 1061.

[9] Braithwaite D. et al., Scaling properties of the anisotropic critical current density in bulk textured YBaCuO. Evidence toward a 3D flux line lattice, $J$ Lon' Temp. Physics 91 (1991) 1 ; see also 92 (1993) 295.

[10] Tournier R., Sulpice H., Lejay P., Chaminade J. P., Pending patent Nº 90/00366 du 5/1/90.

[11] Feinberg D. and Villard C., Intrinsic pinning and Lock-in transition of flux lines in layered type II superconductors, Phys. Rev Lett. 65 (1990) 919.

[12] Lepropre M., Monot I., Delamare M. P., Hervieu M., Simon Ch., Provost J., Desgardin G.. Crvogenics 34 (1994) 63. 